\title{
Judah Folkman, a pioneer in the study of angiogenesis
}

\author{
Domenico Ribatti
}

Received: 4 December 2007/ Accepted: 13 January 2008/Published online: 5 February 2008

(C) Springer Science+Business Media B.V. 2008

\begin{abstract}
More than 30 years ago, Judah Folkman found a revolutionary new way to think about cancer. He postulated that in order to survive and grow, tumors require blood vessels, and that by cutting off that blood supply, a cancer could be starved into remission. What began as a revolutionary approach to cancer has evolved into one of the most exciting areas of scientific inquiry today. Over the years, Folkman and a growing team of researchers have isolated the proteins and unraveled the processes that regulate angiogenesis. Meanwhile, a new generation of angiogenesis research has emerged as well, widening the field into new areas of human disease and deepening it to examine the underlying biological processes responsible for those diseases.
\end{abstract}

Keywords Angiogenesis - Antiangiogenesis ·

History of medicine $\cdot$ Tumor progression

\section{Early evidence of tumor cells releasing specific growth factor for blood vessels}

In 1939, Ide et al. [1] were the first to suggest that tumors release specific factors capable of stimulating the growth of blood vessels. In 1945, Algire and Chalkley [2] were the first to appreciate that growing malignancies could continuously elicit new capillary growth from the host. They used a transparent chamber implanted in a cat's skin to study the vasoproliferative reaction secondary to a wound or implantation of normal or neoplastic tissues. They

\section{Ribatti $(\bowtie)$}

Department of Human Anatomy and Histology, University of

Bari Medical School, Piazza G. Cesare, 11, Policlinico, 70124

Bari, Italy

e-mail: ribatti@anatomia.uniba.it showed that the vasoproliferative response induced by tumor tissues was more substantial and earlier than that induced by normal tissues or following a wound. They concluded that the growth of a tumor is closely connected to the development of an intrinsic vascular network.

In 1956, Melwin and Algire [3] found that the vasoproliferative response of normal or neoplastic tissues transplanted into muscle was not significantly different with respect to the time of onset of new blood vessels, though it was stronger when the implantation was performed in a resection area. In addition, while normal tissues induced a vasoproliferative response confined to the host, tumor tissues induced the formation of neovessels that pierced the implant. Lastly, the intensity of the response seemed to be influenced by the distance between the implant and the host's vessels: normal tissue was unable to induce a response if placed more than $50 \mu \mathrm{m}$ away, whereas tumor tissue had a longer activity range.

In 1968, Greenblatt and Shubik [4] implanted Millipore chambers (pore size $0.45 \mu \mathrm{m}$ ) into a hamster's cheek pouch and placed some tumor fragments around them. In a few days, the growing tumor mass engulfed the whole chamber, whose pores were permeable to the tumor interstitial fluid, but not to the tumor cells. New blood vessels, however, were formed in any case very likely through the release of a diffusible factor that could pass through the pores. Ehrman and Knoth [5] confirmed these data with tumor fragments laid on Millipore filters planted on the chick embryo chorioallantoic membrane (CAM).

\section{Tumors in isolated perfused organs: absence of angiogenesis}

In 1963, Folkman and Becker studied hemoglobin solutions as potential substitutes for blood transfusion. To test 
which solution was optimal for tissue survival, they perfused these solutions through the vasculature of canine thyroid glands, by using an apparatus with a silicone rubber oxygenator. The glands survived for about 2 weeks. They could distinguish different hemoglobin preparations by histologic analysis of the thyroid glands after a week or more of continuous arterial perfusion. To determine if these isolated organs could support growth, they injected them with adult mouse melanoma cells. Tiny tumors developed but stopped growing at 1- to 2-mm diameter and never became vascularized [6]. Endothelial cells swelled and could not proliferate in the presence of free hemoglobin solutions lacking platelets [7]. However, the tumors were not dead. When they were transplanted to their host mice, they rapidly vascularized and grew to more than $1 \mathrm{~cm}^{3}$.

Folkman et al. observed that when tumor cells were inoculated into isolated perfused organs, tumors were limited in size to $1-2 \mathrm{~mm}^{3}$ [6]. Subsequently, they found that neovascularization does not occur in isolated perfused organs and that tumors transplanted from these organs to syngeneic mice became vascularized and grew rapidly to $1-2 \mathrm{~mm}^{3}$. This was the first evidence that the absence of neovascularization correlated with severe restriction of tumor growth.

The data were consistent with work from Harry Green, who had shown long before that growth of rabbit tumors transplanted into the anterior chamber of the guinea pig coincided with the growth of new blood vessels. Tumors that remained viable, but did not grow, had no visible blood vessels [8].

\section{Hypothesis: tumor growth is angiogenesis dependent}

In 1971, Folkman published in the "New England Journal of Medicine" a hypothesis that tumor growth is angiogenesis dependent and that inhibition of angiogenesis could be therapeutic [9]. This article also introduced the term anti-angiogenesis to mean the prevention of new vessel sprout from being recruited by a tumor. The hypothesis predicted that tumors would be enable to grow beyond a microscopic size of $1-2 \mathrm{~mm}^{3}$ without continuous recruitment of new capillary blood vessels.

This concept is now widely accepted because of supporting data from experimental studies and clinical observations carried out over the intervening years.

\section{Evidence that tumors are angiogenesis dependent}

Folkman and collaborators provide evidence for the dependence of tumor growth on neovascularization:
(1) Tumor growth in the avascular cornea proceeds slowly at a linear rate, but after vascularization, tumor growth is exponential [10].

(2) Tumors suspended in the aqueous fluid of the anterior chamber of the rabbit eye and observed for a period up to 6 weeks remain viable, avascular, and of limited size (less than $1 \mathrm{~mm}^{3}$ ) and contain a population of viable and mitotically active tumor cells. These tumors induce neovascularization of the iris vessels but are too remote from these vessels to be invaded by them. After implantation contiguous to the iris, which had abundant blood vessels, the tumors induced neovascularization and grow rapidly, reaching 16,000 times the original size within 2 weeks [11]. This experiment introduced the concept of tumor dormancy brought about by prevention of neovascularization. In a parallel study, tumors were suspended in the aqueous humor of the anterior chamber, placed at various distances from the iris vessels, and compared with tumors implanted directly on the iris and with those implanted in the cornea [12]. Moving the distant, dormant tumors closer to the iris jump started their growth. This suggested that this type of tumor dormancy was caused not by cell cycle arrest or immune control, but by a lack of blood supply.

(3) B-16 mouse melanoma, V-79 Chinese hamster lung cells, and L-5178 Y murine leukemia cells were plated in soft agar [13]. After 6-7 days of incubation, spheroid colonies of $0.1 \mathrm{~mm}$ were visible. All spheroids first enlarged exponentially for a few days and then continued on a linear growth curve for 523 weeks before reaching a diameter beyond which there was no further expansion. This was termed the dormant phase. After the dormant diameter was reached, these spheroids remained viable for 35 months, or as long as they were frequently transferred to new medium. Cells in the periphery of the spheroid incorporated ${ }^{3} \mathrm{H}$-labeled thymidine while cells in the center died. This is a form of population dormancy in which the proliferating cells near the surface of the spheroid just balance those dying cells deep in the center of the spheroid.

(4) Tumors implanted on the CAM of the chick embryo do not exceed a mean diameter of $0.93 \pm 0.29 \mathrm{~mm}$ during the prevascular phase (approximately $72 \mathrm{~h}$ ). Rapid growth begins, however, within $24 \mathrm{~h}$ after vascularization, and tumors reach a mean diameter of $8.0 \pm 2.5 \mathrm{~mm}$ by 7 days [14].

(5) Tumors grown in the vitreous of the rabbit eye remain viable but attain diameters of less than $0.50 \mathrm{~mm}$ for as long as 100 days. Once such a tumor reaches the retinal surface, it becomes neovascularized and within 
2 weeks can undergo a 19,000-fold increase in volume over the avascular tumor [15].

(6) The CAM appears at day 5 during development of the chick embryo. The ${ }^{3} \mathrm{H}$-thymidine labeling index of its vascular endothelium decreases with age, with an abrupt reduction at day 11 [16]. Prior to day 11, labeling index is approximately $23 \%$; during 11 days, the labeling index decreases to $2.8 \%$, and subsequently, the cells begin to acquire the structural characteristics of matured, differentiated endothelium. One-millimeter fragments of fresh Walker 256 carcinoma were implanted on the CAM from day 3 to day 16 [14]. The size of the tumors was measured daily, and the onset of vascularization of each tumor was determined in vivo with a stereomicroscope and confirmed with histological sections. Proliferation of chick capillaries occurred in the neighborhood of the tumor graft by $24 \mathrm{~h}$ after implantation, but capillary sprouts did not penetrate the tumor graft until approximately $72 \mathrm{~h}$. During the avascular phase, tumor diameter did not exceed $1 \mathrm{~mm}$. Small tumor implants of $0.5 \mathrm{~mm}$ or less grew to $1 \mathrm{~mm}$ and stopped expanding. Larger tumor implants of 2- or 3-mm shrank until they reached 1-mm diameter. During the first $24 \mathrm{~h}$ after penetration by capillaries, there was a rapid tumor growth. Neovascularization was not grossly observable with the stereomicroscope until after day 10 or 11 . Tumors implanted on the CAM after day 11 grow at slower rate in parallel with the reduced rates of endothelial growth.

(7) When tumor grafts of increasing size (from 1 to $4 \mathrm{~mm}$ ) are implanted on the 9-day CAM, grafts larger than $1 \mathrm{~mm}$ undergo necrosis and autolysis during the 72-h prevascular phase. They shrink rapidly until the onset of neovascularization, when rapid tumor growth resumes [14]. In another study [17], the behavior of tumor grafts on the CAM was compared to grafts of normal adult and embryonic tissues. In tumor tissue, preexisting blood vessels within the tumor graft disintegrated by $24 \mathrm{~h}$ after implantation. Neovascularization did not occur until after at least 3 days, and only by penetration of proliferating host vessels into the tumor tissue. There was marked neovascularization of host vessels in the neighborhood of the tumor graft. By contrast, in embryonic graft, preexisting vessels did not disintegrated. They reattached by anastomosis to the host vessels within 1-2 days, but with minimal or almost no neovascularization on the part of the host vessels. In adult tissues, the preexisting graft vessels disintegrated; there was no reattachment of their circulation with the host, and adult tissues did not stimulate capillary proliferation. These studies suggest that only tumor grafts are capable of stimulating formation of new blood vessels in the host.

(8) In transgenic mice that develop carcinomas of the $\beta$ cells in the pancreatic islets, large tumors arise only from a subset of preneoplastic hyperplastic islets that have become vascularized [18].

\section{Isolation of the first angiogenic tumor factor}

Until the early 1970s, it was widely assumed that tumors did not produce specific angiogenic proteins. The conventional widsom was that tumor vasculature was an inflammatory reaction to dying or necrotic tumor cells.

Previous studies had shown that tumor-stimulated vessel growth did not require direct contact between tumor and host tissue $[4,5]$. This made sense to Folkman, who reasoned that a soluble factor would be more likely to reach nearly than distant blood vessels. He and his colleagues isolated an angiogenic factor in 1971 [19]. The homogenate of a Walker 256 carcinoma-a breast tumor of SpragueDawley rats-was fractionated by gel-filtration on Sephadex G-100. The fraction that exhibited the strongest angiogenic activity had a molecular weight of about 10,000 daltons and consisted of $25 \%$ RNA, $10 \%$ proteins, and $58 \%$ carbohydrates, plus a possible lipid residue. It was inactivated by digestion with pancreatic ribonuclease, or by heating at $56^{\circ} \mathrm{C}$ for $1 \mathrm{~h}$, and was not modified when kept at $4^{\circ} \mathrm{C}$ for 3 months, nor when treated with trypsin for more than 3 days. This active fraction was subsequently called "tumor angiogenesis factor" (TAF) [19]. Both the cytoplasmic and the nuclear fractions of tumor cells stimulated angiogenesis. In the nuclear fraction, this was found to be associated with nonhistonic proteins [20]. Tumor angiogenesis factor has since been nondestructively extracted from several tumor cell lines, and several low molecular weight angiogenic factors have been isolated, again from the Walker 256 carcinoma. These factors induced a vasoproliferative response in vivo when tested on rabbit cornea or chick CAM, and in vitro on cultured endothelial cells [21-23].

\section{First evidence of the existence of the avascular and vascular phases of solid tumor growth}

The earliest evidence of the existence of the two phases was obtained by Folkman and collaborators in 1963, who perfused the lobe of a thyroid gland with plasma and inoculated a suspension of melanoma B16 tumor cells through the perfusion fluid. These cells grew into small, clearly visible black nodules. The nodules did not exceed 
$1 \mathrm{~mm}$ in diameter and did not connect with the host's vascular network. Their outer third generally remained vital, while the interior portion underwent necrosis. Reimplanted nodules, on the other hand, equipped themselves with a vascular network and grew very rapidly. The conclusion was thus drawn that the absence of vascularization limits the growth of solid tumors.

Further research by Folkman's group resulted in an experimental system in which the tumor, or its extracts, could be separated from the vascular bed [24, 25]. This system was based on subcutaneous insufflation to lift the skin of a rat and form a poorly vascularized region below it. Millipore filters containing Walker 256 cancer cells or their cytoplasmic or nuclear extracts (TAF) were implanted into the fascial floor of the dorsal air sac. At intervals thereafter, ${ }^{3} \mathrm{H}$-labeled thymidine was injected into the air sac, and the tissues were examined by autoradiography and electron microscopy. Autoradiographs showed thymidine- ${ }^{3} \mathrm{H}$ labeling in endothelial cells of small vessels, 1$3 \mathrm{~mm}$ from the site of implantation, as early as 6-8 $\mathrm{h}$ after exposure to tumor cells. DNA synthesis by endothelium subsequently increased, and within 48 h new blood vessels formation was detected. The presence of labeled endothelial nuclei, endothelial mitosis, and regenerating endothelium was confirmed by electron microscopy. Tumor angiogenesis factor also induced neovascularization and endothelial DNA synthesis after 48 h. Further ultrastructural autoradiographic studies were carried out with the same model [25]. It was apparent that by $48 \mathrm{~h}$ there was ultrastructural evidence of regenerating endothelium, including marked increase in ribosomes and endoplasmic reticulum, scarce or absent pinocytotic vesicles, and discontinuous basement membrane. Labeled endothelial cells were seen along newly formed sprouts as well as in parent vessels. Furthermore, pericytes were also shown to synthesize DNA.

In another series of experiments, 1-mm fragments from Brown-Pearce and V2 carcinomas were implanted into the avascular stroma of a rabbit cornea 1-6 $\mathrm{mm}$ away from the limbic vessels, and observed the tumor growth daily with a stereomicroscope [10]. After 1 week, new blood vessels had invaded the cornea starting from the edge closer to the site of implantation and developed in that direction at $0.2 \mathrm{~mm}$ and then about $1 \mathrm{~mm} /$ day. Once the vessels reached the tumor, it grew very rapidly to permeate the entire globe within 4 weeks.

\section{Dormancy of micrometastases may be governed by angiogenesis}

Folkman and collaborators found that metastases were suppressed when a primary tumor was implanted and allowed to grow in nude mice, whereas they underwent neovascularization and became clinically evident when primary neoplasm was removed. In the absence of angiogenesis, micrometastases rarely exceeded $0.2-\mathrm{mm}$ diameter and contained many proliferating tumor cells balanced by many apoptotic cells. When they were allowed to become angiogenic, they grew rapidly. Dormancy may be generalizable to a variety of tumors in which blocked angiogenesis results in balanced tumor cell proliferation and apoptosis [26].

\section{Prognostic significance of tumor vascularity}

In 1972, Brem in the Folkman laboratory reported the first quantitative method for histologic grading of tumor angiogenesis. He correlated neovascularization in human brain tumors with tumor grade [27]. In the early 1990s, Weidner and collaborators [28-30] showed that measurement of microvascular density within isolated regions of high vessel concentration (i.e., hotspots) was a prognostic indicator for human breast and prostate carcinomas.

Microvascular density counting protocols have become the morphological gold standard to assess the neovasculature in human tumors. This method requires the use of specific markers to vascular endothelium and of immunohistochemical procedures to visualize microvessels. Microvascular density determined in primary tumors is significantly associated with metastasis and prognosis in several solid and hematological tumors.

\section{Antiangiogenesis}

The existence of specific angiogenesis inhibitors was first postulated by Folkman in 1971 in an editorial. No angiogenesis inhibitors existed before 1980, and few scientists thought at that time that such molecules would ever be found.

From 1980 to 2005, Folkman's laboratory reported the discovery of 12 angiogenesis inhibitors (Table 1). The first angiogenesis inhibitor was found in cartilage, an avascular tissue that resists invasion by many tumors [31]. Brem and Folkman demonstrated that tumor-induced vessels were inhibited by a diffusible factor from neonatal rabbit cartilage [32]. The partially purified inhibitor suppressed tumor growth when it was infused into the vascular bed of murine and rabbit tumors [33]. 2-methoxyestradiol was first reported by Fotsis et al. [34], and the article reporting 2-methoxyoestradiol and its molecular mechanism as an inhibitor of tubulin polymerization by acting at the colchicine site was published a month later [35]. 
Table 1 Angiogenesis inhibitors discovered in Folkman's laboratory from 1980 to 2005

\begin{tabular}{l} 
1980. Interferon alpha-beta \\
1982. Platelet factor 4/ \\
protamine \\
1985. Angiostatic steroids \\
1990. Fumagillin \\
1994. Angiostatin \\
1994. Thalidomide \\
1994. 2-methoxyestradiol \\
1997. Endostatin \\
1999. Cleaved antithrombin III \\
2002. 3-Aminotholidomide \\
2003. DBF-maf \\
2005. Caplostatin \\
\hline
\end{tabular}

\section{Interferon alpha}

Interferon alpha was first shown to inhibit endothelial cell migration in a dose-dependent and reversible manner in 1980 by Zetter in Folkman's laboratory [36]. Since 1988, interferon alpha has been used successfully to cause complete and durable regression of life-threatening pulmonary hemangiomatosis, hemangiomatosis of the brain, airway and liver in infants, recurrent high-grade giant cell tumors refractory to conventional therapy, and angioblastomas [37-39].

A 12-year-old boy with fatal pulmonary hemangiomatosis had a complete remission and recovered completely after 7 months of therapy with interferon alpha. Therapy was continued for 7 years. This led to the successful use of low-dose daily interferon alpha therapy administered subcutaneously to infants with sight-threatening or lifethreatening hemangiomas and hemangioendotheliomas of the heart, airway, and liver [37-39].

\section{Platelet factor 4/protamine}

Protamine was shown to be an angiogenesis inhibitor [40], but cumulative toxicity from prolonged administration and a narrow window of angiostatic efficacy prevented its consideration for clinical use.

Platelet factor 4 was first tested for antiangiogenic activity because its method of binding and neutralizing heparin is similar to that of protamine [40]. Recombinant human platelet factor 4 (rHuPF4) has been produced [41]. It specifically inhibited endothelial proliferation and migration in vitro [42]. The inhibitory activities are associated with the carboxy-terminal region of the molecule. The growth of human colon carcinoma in athymic mice, as well as the growth of murine melanoma, was markedly inhibited by intralesional injections, whereas tumor cells were completely insensitive to rHuPF4 in vitro at levels that inhibited normal endothelial cell proliferation. Systemic administration of rHuPF4 has so far been ineffective against tumor growth, perhaps because of rapid inactivation or clearance of the peptide.

\section{Angiostatic steroids}

Folkman had begun to use the CAM of the chick embryo to detect angiogenic activity in fractions being purified from tumor extracts. The addition of heparin increased the speed of development of the angiogenic reaction so that it could be read 1-2 days later [40]. But one problem with this assay is that occasionally eggshell dust falls on the CAM and causes background inflammation. Folkman guessed that adding cortisone to the CAM might eliminate the irritation from the shell dust but not abolish the tumorangiogenic reaction. As expected, cortisone alone prevented shell dust inflammation without interfering with angiogenesis induced by tumor extracts. The surprise was that when heparin and cortisone were added together, tumor angiogenesis was inhibited [43]. Furthermore, when this combination of heparin and steroid was suspended in a methylcellulose disk and implanted on the young (6-day) CAM, growing capillaries regressed leaving in their place, $48 \mathrm{~h}$ later, an avascular zone. The antiangiogenic effect was specific for growing capillaries. Mature nongrowing capillaries in older membranes were unaffected. Nonanticoagulant heparin had the same effect. A hexasaccharide fragment with a molecular weight of approximately 16,000 was found to be the most potent inhibitor of angiogenesis (in the presence of a corticosteroid). The combination of the heparin hexasaccharide fragment and cortisone also inhibited tumor-induced angiogenesis in the rabbit cornea.

The regression of a growing vessel exposed to heparinsteroid combinations begins with endothelial cell rounding and is followed by cessation of endothelial proliferation, desquamation of endothelial cells, and retraction of the capillary sprout [44]. These events occur as 24-48 h and are accompanied by dissolution of the basement membrane of the new capillary vessels.

\section{Fumagillin}

Fumagillin was found by Ingber in the Folkman laboratory to inhibit endothelial cell proliferation without causing endothelial cell apoptosis, when a tissue culture plate of endothelial cells became contaminated with a fungus Aspergillus fumigatus fresenius [45]. A conditioned medium from fungal cultures contained an inhibitor of endothelial cell proliferation and angiogenesis, which, upon purification, was found to be fumagillin, a polyene macrolide. When capillary endothelial cells were stimulated by fibroblast growth factor-2 (FGF-2), half-maximal inhibition 
was observed with fumagillin at $100 \mathrm{pg} / \mathrm{ml}$. This antiproliferative effect appeared to be relatively specific for endothelial cells because inhibition of nonendothelial cells, including tumor cells, was observed at up to 1,000 -fold higher concentrations.

Scientists at Takeda Chemical Industries (Osaka, Japan) made a synthetic analogue of fumagillin, called TNP-470, which inhibits endothelial proliferation in vitro at a concentration 3 logs lower than the concentration necessary to inhibit fibroblasts, and tumor cells.

\section{Angiostatin and endostatin}

They were discovered by M. O'Reilly in Folkman laboratory based on Folkman's hypothesis of a mechanism to explain the phenomenon that surgical removal of certain tumors leads to rapid growth of remote metastases. This hypothesis said that if tumors produce both stimulators and inhibitors of angiogenesis, an excess of inhibitors could accumulate within an angiogenic tumor. In the circulation however, the ratio would be reversed. Angiogenesis inhibitors would increase relative to stimulators, because of rapid clearance of stimulators from the blood. Folkman formulated this hypothesis after reading Bouck's first report in 1989 that the emergence of tumor angiogenesis was the result of a shift in balance between positive and negative regulators of angiogenesis in a tumor [46]. Bouck reported that the switch to angiogenesis during tumorigenesis of transformed hamster cells was associated with downregulation of an inhibitor of angiogenesis, thrombospondin. She suggested that the switch to the angiogenic phenotype could be the result of a shift in the net balance of positive and negative regulators of angiogenesis.

In 1991, O'Reilly began to screen a variety of transplantable murine tumors for their ability to suppress metastases. A Lewis lung carcinoma was the most efficient. When the metastasis-suppressing primary tumor was present in the dorsal subcutaneous position, microscopic lung metastases remained dormant at a diameter of less than $200 \mu \mathrm{m}$ surrounding a preexisting microvessel, but revealed no new vessels. Within 5 days after surgical removal of the primary tumor, lung metastases became highly angiogenic and grew rapidly, killing their host by 15 days [47]. This striking evidence that primary tumor could suppress angiogenesis in its secondary metastases by a circulating inhibitor was further supported by the demonstration that a primary tumor could also suppress corneal angiogenesis by an implanted pellet of FGF-2. O'Reilly then succeeded in purifying this inhibitor from the serum and urine of tumor-bearing animals. It was a $38-\mathrm{kD}$ internal fragment identical in amino acid sequence to the first four kringle structures of plasminogen and it was named angiostatin. Angiostatin specifically inhibited the proliferation of growing vascular endothelial cells and had no effect on resting confluent endothelial cells or on other cell types, including smooth muscle cells, epithelial cells, fibroblasts and tumor cells. It also inhibited growth of primary tumors by up to $98 \%$ [48] and was able to induce regression of large tumors (1-2\% of body weight) and maintain them at a microscopic dormant size.

Based on the same rationale and strategy, O'Reilly isolated and purified another angiogenesis inhibitor from a murine hemangioendothelioma. This inhibitor, called endostatin [49], is a $20-\mathrm{kD}$ protein with an N-terminal amino acid sequence identical to the carboxyterminus of collagen XVIII. It was purified directly from tumor cellconditioned medium. Endostatin is also a specific inhibitor of endothelial proliferation and has no effects on resting endothelial cells or on other cell types. It is slightly more potent than angiostatin and also causes regression of large tumors to a microscopic size.

\section{Thalidomide}

In 1994, D'Amato in the Folkman laboratory reported that thalidomide is an angiogenic inhibitor [50]. Corneal neovascularization in rabbits induced by FGF-2 or VEGF was blocked by orally administered thalidomide. This activity of thalidomide was mainly the result of its direct effect on inhibiting new blood vessel formation and not on suppression of infiltrating host inflammatory cells. Histologic sections of the pretreated neovascularized corneas were virtually free of inflammatory cells. Thalidomide also inhibited corneal neovascularization in mice, but it was necessary to give the drug by the intraperitoneal route and at high doses, because mice do not metabolize thalidomide effectively.

\section{2-Methoxyestradiol}

In 1994, D'Amato in Folkman laboratory demonstrated that a metabolite of estradiol, 2-methoxyestradiol, inhibited angiogenesis in the chick CAM [35]. Moreover, since 2methoxyestradiol causes mitotic perturbations, they examined its interactions with tubulin and showed that 2methoxyestradiol bound to colchicine site of tubulin and, depending on reaction conditions, either inhibited assembly or seems to be incorporated into a polymer with altered stability properties.

\section{Cleaved antithrombin III}

A human small-cell lung carcinoma suppressed angiogenesis and tumor growth at remote sites in immunodeficient 
mice. These cells generated an enzyme in vitro that converted the $58-\mathrm{kD}$ conformation of circulating antithrombin III to a 53-kD form of the protein [51] in which the externally configured stressed loop of antithrombin was retracted into the body of the molecule. The $53-\mathrm{kD}$ form is a specific endothelial inhibitor and a potent angiogenesis inhibitor and has no thrombin binding activity.

\section{Caplostatin}

Caplostatin is a nontoxic synthetic analogue of fumagillin conjugated to a water-soluble- $N$-(2-hydroxypropyl)methacrylamide (HPMA) copolymer [52, 53]. Caplostatin has a similar broad antitumor spectrum of TNP-470 without any toxicity. In addition to its antiangiogenic activity, caplostatin is the most potent known inhibitor of vascular permeability [53]. Caplostatin prevents vascular leakage induced by VEGF, bradykinin, histamine, and plateletactivating factor and prevents pulmonary edema induced by interleukin- 2 .

\section{Antiangiogenic chemotherapy}

Browder in the Folkman laboratory was the first to demonstrate the concept that by optimizing the dosing schedule of conventional cytotoxic chemotherapy to achieve more sustained apoptosis of endothelial cells in the vascular bed of a tumor, it is possible to achieve more effective control of tumor growth in mice, even if the tumor cells are drug resistant [54]. Conventional chemotherapy is administered at maximum tolerated doses followed by off-therapy intervals of 2-3 weeks to allow the bone marrow and gastrointestinal tract to recover. In contrast, antiangiogenic chemotherapy is administered more frequently at lower doses, without long interruptions in therapy and with little or no toxicity. During antiangiogenic chemotherapy, endothelial cell apoptosis and capillary dropout precede the death of tumor cells that surround each capillary.

\section{Concluding remarks}

Currently, several compounds with angiostatic activity are approved for clinical use, and many are in late-stage clinical development. However, the results from clinical trials have not shown the antitumor effects which were expected following preclinical studies. It appears that clinical applications of antiangiogenic therapy are more complex than originally thought.

The main problem in the development of antiangiogenic agents is that multiple angiogenic molecules may be produced by tumors, and tumors at different stages of development may depend on different angiogenic factors for their blood supply. Therefore, blocking a single angiogenic molecule was expected to have little or no impact on tumor growth. Current development of targeted antiangiogenic agents include their use in adjuvant settings and the combination of different antiangiogenic inhibitors to take a more comprehensive approach in blocking tumor angiogenesis.

Advancing insights into fundamental mechanisms will be necessary in the development of novel anticancer strategies based on inhibition of angiogenesis.

Acknowledgments The author is grateful to Prof. Judah Folkman for his suggestion and critical reading of the manuscript. This work was supported by grants from AIRC (Italian Association for Cancer Research, Regional Funds), MIUR (Italian Ministry of University and Research) (FIRB 2001, PRIN 2005 and CARSO Consortium Project 72/2).

\section{References}

1. Ide AG, Baker NH, Warren SL (1939) Vascularization of the Brown-Pearce rabbit epithelioma transplant as seen in the transparent ear chamber. AJR 32:891-899

2. Algire GH, Chalkley HW (1945) Vascular reactions of normal and malignant tissue in vivo. J Natl Cancer Inst 6:73-85

3. Melwin RM, Algire GH (1956) The role of graft and host vessels in vascularization of grafts of normal and neoplastic tissues. $J$ Natl Cancer Inst 17:23-33

4. Greenblatt M, Shubik P (1968) Tumor angiogenesis: transfilter diffusion studied in the hamster by the transparent chamber technique. J Natl Cancer Inst 41:1111-1124

5. Ehrman RL, Knoth M (1968) Choriocarcinoma: transfilter stimulation of vasoproliferation in the hamster cheek pouch studied by light and electron microscopy. J Natl Cancer Inst 41:1329-1341

6. Folkman MJ, Long DM, Becker FF (1963) Growth and metastasis of tumor in organ culture. Cancer 16:453-467

7. Gimbrone MA Jr, Aster RH, Cotran RS, Corkery J, Jandl JH, Folkman J (1969) Preservation of vascular integrity in organs perfused in vitro with a platelet-rich medium. Nature 222:33-36

8. Green HSN (1941) Heterologous transplantation of mammalian tumors: I. The transfer of rabbit tumors to alien species. J Exp Med 73:461-474

9. Folkman J (1971) Tumor angiogenesis. Therapeutic implications. N Engl J Med 285:1182-1186

10. Gimbrone MA Jr, Cotran RS, Folkman J (1974) Tumor growth and neovascularization: an experimental model using rabbit cornea. J Natl Cancer Inst 52:413-427

11. Gimbrone MA, Leapman SB, Cotran RS, Folkman J (1972) Tumor dormancy in vivo by prevention of neovascularization. $\mathrm{J}$ Exp Med 136:261-276

12. Gimbrone MA, Leapman S, Cotran RS, Folkman J (1973) Tumor angiogenesis: iris neovascularization at a distance from experimental intraocular tumors. J Natl Cancer Inst 50:219-228

13. Folkman J, Hochberg M (1983) Self-regulation of growth in three dimensions. J Exp Med 138:745-753

14. Knighton D, Ausprunk D, Tapper D, Folkman J (1977) Avascular and vascular phases of tumour growth in the chick embryo. Br J Cancer 35:347-356

15. Brem S, Brem H, Folkman J, Finkelstein D, Patz G (1976) Prolonged tumor dormancy by prevention of neovascularization in the vitreous. Cancer Res 36:2807-2812 
16. Ausprunk DH, Knighton DR, Folkman J (1974) Differentiation of vascular endothelium in the chick chorioallantois: a structural and autoradiographic study. Dev Biol 38:237-248

17. Ausprunk DH, Knighton DR, Folkman J (1975) Vascularization of normal and neoplastic tissues grafted to the chick chorioallantois. Role of host and preexisting graft blood vessels. Am J Pathol 79:597-618

18. Folkman J, Watson K, Ingber D, Hanahan D (1989) Induction of angiogenesis during the transition from hyperplasia to neoplasia. Nature 339:58-61

19. Folkman J, Merler E, Abernathy C, Williams G (1971) Isolation of a tumor fraction responsible for angiogenesis. J Exp Med 133:275-288

20. Tuan D, Smith S, Folkman J, Merler E (1973) Isolation of the nonhistone proteins of rat Walker 256 carcinoma. Their association with tumor angiogenesis. Biochemistry 12:3159-3165

21. Mc Auslan BR, Hoffman H (1979) Endothelium stimulating factor from Walker carcinoma cells. Relation to tumor angiogenic factor. Exp Cell Res 119:181-190

22. Weiss JB, Brown RA, Kumar S, Phillips P (1979) An angiogenic factor isolated from tumours: a potent low-molecular weight compound. Br J Cancer 40:493-496

23. Fenselau A, Kaiser D, Wallis K (1981) Nucleoside requirements for the in vivo growth of bovine aortic endothelial cells. J Cell Physiol 108:375-384

24. Cavallo T, Sade R, Folkman J, Cotran RS (1972) Tumor angiogenesis: rapid induction of endothelial mitosis demonstrated by autoradiography. J Cell Biol 54:408-420

25. Cavallo T, Sade R, Folkman J, Cotran RS (1973) Ultrastructural autoradiographic studies of the early vasoproliferative response in tumor angiogenesis. Am J Pathol 70:345-362

26. Holmgren L, O'Reilly MS, Folkman J (1995) Dormancy of micrometastases: balanced proliferation and apoptosis in the presence of angiogenesis suppression. Nat Med 1:149-153

27. Brem S, Cotran R, Folkman J (1972) Tumor angiogenesis: a quantitative method for histologic grading. J Natl Cancer Inst 48:347-356

28. Weidner N, Semple JP, Welch WR, Folkman J (1971) Tumor angiogenesis and metastasis-correlation in invasive breast carcinoma. N Engl J Med 324:1-8

29. Weidner N, Folkman J, Pozza F, Bevilacqua P, Allred EN, Moore DH, Meli S, Gasparini G (1992) Tumor angiogenesis: a new significant and independent prognostic indicator in early-stage breast cancer. J Natl Cancer Inst 84:1875-1887

30. Weidner N, Carroll PR, Flax J, Blumenfeld W, Folkman J (1993) Tumor angiogenesis correlates with metastasis in invasive prostate carcinoma. Am J Pathol 143:401-409

31. Eisenstein R, Sorgente N, Soble L, Miller A, Kuettner KE (1973) The resistance of certain tissues to invasion: penetrability of explanted tissues by vascularized mesenchyme. Am J Pathol 73:765-774

32. Brem H, Folkman J (1975) Inhibition of tumor angiogenesis mediated by cartilage. J Exp Med 141:427-439

33. Langer R, Conn H, Vacanti J, Haudenschild C, Folkman J (1980) Control of tumor growth in animals by infusion of an angiogenesis inhibitor. Proc Natl Acad Sci USA 77:4331-4335

34. Fotsis T, Zhang Y, Pepper MS, Adlercreutz H, Montesano R, Nawroth PP, Schweigerer L (1994) The endogenous oestrogen metabolite 2-methoxyoestradiol inhibits angiogenesis and suppresses tumor growth. Nature 368:237-239

35. D’Amato RJ, Lin CM, Flynn E, Folkman J, Hamel E (1994) 2Methoxyestradiol, an endogenous mammalian metabolite, inhibits tubulin polymerization by interacting at the colchicine site. Proc Natl Acad Sci USA 91:3964-3968

36. Brouty-Boye D, Zetter BR (1980) Inhibition of cell motility by interferon. Science 208:516-518
37. Ezekowitz RA, Mulliken JB, Folkman J (1972) Interferon alfa-2a therapy for lifethreatening hemangiomas of infancy. New Engl $J$ Med 326:1456-1463

38. Kaban LB, Mulliken JB, Ezekowitz RA, Ebb D, Smith PS, Folkman J (1999) Antiangiogenic therapy of a recurrent giant cell tumor of the mandible with interferon alfa-2a. Pediatrics 103:1145-1149

39. Folkman J (2002) Discussion: antiangiogenic therapy with interferon alpha for giant lesions of the jaws. J Oral Maxillofac Surg 60:1111-1113

40. Taylor S, Folkman J (1972) Protamine is an inhibitor of angiogenesis. Nature 297:307-312

41. Maione TE, Gray GS, Petro J, Hunt AJ, Donner AL, Bauer SI, Carson HF, Sharpe RJ (1990) Inhibition of angiogenesis by recombinant human platelet factor- 4 and related peptides. Science 247:71-79

42. Sharpe RJ, Byers HR, Scott CF, Bauer SI, Maione TE (1990) Growth inhibition of murine melanoma and human colon carcinoma by recombinant human platelet factor 4 . J Natl Cancer Inst 82:848-853

43. Folkman J, Langer R, Linhardt R, Haudenschild C, Taylor S (1983) Angiogenesis inhibition and tumor regression caused by heparin or a heparin fragment in the presence of cortisone. Science 221:719-725

44. Ingber DE, Madri JA, Folkman J (1986) A possible mechanism for inhibition of angiogenesis by angiostatic steroids: induction of capillary basement membrane dissolution. Endocrinology 119:1768-1775

45. Ingber D, Fujita T, Kishimoto S, Sudo K, Kanamaru T, Brem H, Folkman J (1990) Synthetic analogues of fumagillin that inhibit angiogenesis and suppress tumour growth. Nature 348: 355-357

46. Rastinejad F, Polverini PJ, Bouck NP (1989) Regulation of the activity of a new inhibitor of angiogenesis by a cancer suppressor gene. Cell 56:345-355

47. O'Reilly MS, Holmgren L, Shing Y, Chen C, Rosenthal RA, Moses M, Lane WS, Cao Y, Sage EH, Folkman J (1994) Angiostatin: a novel angiogenesis inhibitor that mediates the suppression of metastasis by a Lewis lung carcinoma. Cell 79:315-328

48. O'Reilly MS, Holmgren L, Chen C, Folkman J (1996) Angiostatin induces and sustain dormancy of human primary tumors in mice. Nat Med 2:689-692

49. O'Reilly MS, Boehm T, Shing Y, Fukai N, Vasios G, Lane WS, Flynn E, Birkhead JR, Olsen BR, Folkman J (1997) Endostatin: an endogenous inhibitor of angiogenesis and tumor growth. Cell 88:277-285

50. D’Amato RJ, Loughnan MS, Flynn E, Folkman J (1994) Thalidomide is an inhibitor of angiogenesis. Proc Natl Acad Sci USA 91:4082-4085

51. O'Reilly MS, Pirie-Shepherd S, Lane WS, Folkman J (1999) Antiangiogenic activity of the cleaved conformation of the serpin antithrombin. Science 285:1926-1928

52. Satchi-Fainaro R, Puder M, Davies JW, Tran HT, Sampson DA, Greene AK, Corfas G, Folkman J (2004) Targeting angiogenesis with a conjugate of HPMA copolymer and TNP-470. Nat Med 10:255-261

53. Satchi-Fainaro R, Mamluk R, Wang L, Short SM, Nagy JA, Feng D, Dvorak AM, Dvorak HF, Puder M, Mukhopadahyay D, Folkman J (2005) Inhibition of vessel permeability by TNP-470 and its polymer conjugate, caplostatin. Cancer Cell 7: 251-261

54. Browder T, Butterfield CE, Kraling BM, Shi B, Marshall B, O'Reilly MS, Folkman J (2000) Antiangiogenic scheduling of chemotherapy improves efficacy against experimental drugresistant cancer. Cancer Res 60:1878-1886 